\title{
O perfil das consultas clínicas em consultórios farmacêuticos: uma revisão
}

\section{integrativa de literatura}

\author{
The profile of clinical consultations in pharmaceutical offices: an integrative literature review \\ El perfil de las consultas clínicas en las oficinas farmacéuticas: una revisión integradora de la \\ literatura
}

Recebido: 04/10/2021 | Revisado: 10/10/2021 | Aceito: 15/10/2021 | Publicado: 18/10/2021

\author{
Rafael Galvan Luiz \\ ORCID: https://orcid.org/0000-0001-6680-6152 \\ Faculdade Integrada Carajás, Brasil \\ E-mail: rafaelgalvan2luiz@gmail.com \\ Thalia Rola Lima \\ ORCID: https://orcid.org/0000-0002-6259-9691 \\ Faculdade Integrada Carajás, Brasil \\ E-mail: thaliarolalima@gmail.com \\ Carolinne de Oliveira Marquez \\ ORCID: https://orcid.org/0000-0001-6556-5094 \\ Faculdade Integrada Carajás, Brasil \\ E-mail: carolzinhaoliveiramarquez@yahoo.com.br
}

\begin{abstract}
Resumo
Atualmente os consultórios farmacêuticos assumem um papel relevante para o atendimento à população e diante disso as consultas clínicas vem ganhando espaço sendo um dos meios mais acessíveis de cuidado à saúde evitando assim a sobrecarga nos hospitais contribuindo para a prevenção à doenças e o uso racional de medicamentos, os farmacêuticos diante dessa realidade se mostram fundamentais no cuidado ao paciente na prestação a atenção e na assistência farmacêutica. Este estudo objetiva analisar o impacto das intervenções farmacêuticas em consultórios e seu parecer durante a consulta evidenciando os efeitos sobre a saúde dos pacientes em relação ao estudo e acompanhamento da farmacoterapia. Trata - se de uma revisão integrativa de literatura onde foram consultadas bases de dados Google Acadêmico e Biblioteca Virtual da Saúde utilizando os descritores: Consultório farmacêutico; Consulta clínica; Farmacêutico clínico, essas análises foram feitas no período de 2016 à 2021 consultando dissertações, teses e trabalhos de conclusão de curso. A maioria dos artigos foi publicada no ano de 2018. O perfil das consultas clínicas tem avançado e continuará em ascensão cada dia mais devido o alcance bem sucedido do paciente ao consultório onde o farmacêutico encontra meios para otimizar a qualidade de vida do paciente em relação à análise e acompanhamento da farmacoterapia prescrita, na atenção farmacêutica em relação aos MIP's (Medicamentos Isentos de Prescrições) que na maioria das vezes são usados indiscriminadamente levando o paciente a gerar PRM's (Problemas Relacionados aos Medicamentos) bem como nas terapias não farmacológicas.
\end{abstract}

Palavras-chave: Consultório farmacêutico; Consulta clínica; Farmacêutico clínico.

\begin{abstract}
Currently, pharmaceutical offices play a relevant role in serving the population and, in view of this, clinical consultations have been gaining space as one of the most accessible means of health care, thus avoiding the overload in hospitals, contributing to the prevention of diseases and the rational use of medicines, in view of this reality, pharmacists are fundamental in patient care in providing care and pharmaceutical assistance. This study aims to analyze the impact of pharmaceutical interventions in offices and their opinion during the consultation, highlighting the effects on patients' health in relation to the study and monitoring of pharmacotherapy. This is an integrative literature review where Google Academic databases and Virtual Health Library were consulted using the descriptors: Pharmaceutical office; Clinical consultation; As a clinical pharmacist, these analyzes were carried out in the period from 2016 to 2021, consulting dissertations, theses and course completion papers. Most articles were published in 2018. The profile of clinical consultations has advanced and will continue to rise more ans more due to the successful reach of patient to the office where the pharmacist finds ways to optimize the patient's quality of life in relation to analysis and monitoring of prescribed pharmacotherapy, in pharmaceutical care in relation to MIP's (Exempt from Prescription Drugs) which are most often used indiscriminately, leading the patient to generate PRM's (Problems Related to Drugs) as well as in non-pharmacological therapies.
\end{abstract}

Keywords: Pharmaceutical office; Clinical consultation; Clinical pharmacist. 


\begin{abstract}
Resumen
En la actualidad, los consultorios farmacéuticos juegan un papel relevante en la atención a la población y, por ello, las consultas clínicas han ido ganado espacio como uno de los medios más accesibles de atención de la salud, evitando así la sobrecarga en los hospitales, contribuyendo a la prevención de enfermedades y el uso racional de los medicamentos, ante esta realidad, los farmacéuticos son fundamentales en la atención al paciente en la prestación asistencial y asistencial farmacéutica. Este estudio tiene como objetivo analizar el impacto de las intervenciones farmacéuticas en consultorios y su opinión durante la consulta, destacando los efectos en la salud de los pacientes em relación al estudio y seguimiento de la farmacoterapia. Se trata de una revisión integradora de la literatura donde se consultaron las bases de datos académicas de Google y la Biblioteca Virtual en Salud utilizando los descriptores: Oficina farmacéutica; Consulta clínica; Como farmacéutico clínico, estos análisis se realizaron en el período de 2016 a 2021, consultando disertaciones, tesis y trabajos de finalización de curso. La mayoría de los artículos fueron publicados en 2018. El perfil de las consultas clínicas ha avanzado y seguirá aumentando cada vez más debido al exitoso alcance del paciente al consultorio donde el farmacéutico encuentra formas de optimizar la calidad de vida del paciente en relación al análisis y seguimiento de la farmacoterapia prescrita, en la atención farmacéutica en relación a los MIP's (Exentos de Medicamentos con Receta) que se utilizan con mayor frecuencia de manera indiscriminada, llevando al paciente a generar PRM's (Problemas Relacionados con los Medicamentos) así como en terapias no farmacológicas.
\end{abstract}

Palabras clave: Oficina farmacéutica; Consulta clínica; Farmacéutico clínico.

\title{
1. Introdução
}

A consulta farmacêutica com acompanhamento da farmacoterapia pode ser uma fonte de informações para pacientes, médicos e equipe multiprofissional de saúde, o farmacêutico tem a oportunidade de instigar direta ou indiretamente o paciente devendo intervir no processo saúde/doença apresentando alternativas para o uso de terapias com medicamentos e desenvolvendo seu trabalho na produção de cuidado às pessoas (Carvalho, 2017).

Em uma sala, o consultório farmacêutico, é o local onde se atribui ao profissional a realização da consulta farmacêutica, sendo garantido nesse espaço um atendimento com privacidade ao paciente, possibilitando assim que o farmacêutico atue mediante exercício legal da profissão (Melgarejo, 2018).

A prescrição farmacêutica através de consultório é um momento histórico para a profissão trazendo à tona o papel do farmacêutico para toda a população, o farmacêutico não é mais um indicador de medicamentos é um profissional que auxilia na expectativa de tratamento (Lopes, 2017)

A assistência farmacêutica trata-se de um conjunto de ações, voltadas ao exercício, proteção e recuperação da saúde, tendo o uso de medicamento como insumo essencial para a recuperação do paciente (Mesquita, 2017). Esse estudo requer a união consciente entre o farmacêutico, paciente e os demais profissionais de saúde. Tal atitude irá proporcionar uma postura saudável e o cuidado para que haja a prevenção de doenças.

O profissional de saúde deve identificar durante a atenção farmacêutica, os conhecimentos, a experiência prática, vivência social e familiar dos pacientes, a fim de promover educação em saúde para o uso racional de medicamento (Silva Júnior, 2019).

O objetivo do perfil das consultas clínicas em consultórios farmacêuticos com assistência farmacêutica é a promoção, proteção e recuperação da saúde do paciente. Um dos passos a ser realizado para alcançar objetivo é avaliar os impactos das intervenções farmacêuticas nas farmácias e determinar o posicionamento dos profissionais em relação a consulta clínica.

\section{O consultório farmacêutico e a consulta farmacêutica}

O consultório trata-se de um espaço reservado dentro da drogaria e ou farmácia para que o farmacêutico possa realizar a anamnese sobre o que enfermo está ingerindo, orientar e ouvir os pacientes com relação à forma de tomar os medicamentos e seu processo de mudança e adaptação clínica (de Paiva, 2021). 
Em um consultório os cuidados farmacêuticos são realizados com pacientes que podem apresentar variados problemas de saúde (Melgarejo, 2019). Os pacientes polimedicados demandam uma atenção extrema diante da tomada de medicamentos e a consulta farmacêutica com acompanhamento da farmacoterapia é indispensável na otimização desse cuidado (ROVER, 2017).

A profissão farmacêutica está mudando de simples oferta de medicamentos para uma função clínica de cuidado ao paciente (Vasconcelos, 2016). O farmacêutico deixou de ser um simples dispensador de medicamentos para assumir a postura clínica dentro da drogaria que é antes de mais nada um estabelecimento de saúde (BOUÇAS, 2017).

Apesar do termo "farmácia clínica" existir desde a década de 1960, no Brasil esta ideia ainda se encontra em desenvolvimento (Reis, 2018). O atendimento clínico nos dias atuais está em pleno desenvolvimento pois está resgatando os primórdios em que o farmacêutico realizava todo o atendimento ao paciente desde à consulta até a atenção farmacêutica e esse resgate influencia muito na confiança que se estabelece entre o farmacêutico e o paciente.

Devido à acessibilidade ao paciente, o farmacêutico exerce um papel muito importante nas informações prestadas. Os serviços farmacêuticos clínicos tendem a ser mais efetivos em pacientes que apresentam maior tendência para a ocorrência de problemas da farmacoterapia (Silva, 2018). A adesão ao tratamento é uma das grandes dificuldades para o sucesso da farmacoterapia, diante disso a consulta farmacêutica se faz necessária para que o paciente adquira qualidade de vida (CANTO, 2016).

A consulta farmacêutica é considerada hoje, uma porta de entrada para fidelizar o serviço com o paciente, promovendo o vínculo e obtendo resultados muito positivos, tanto para a gestão, quanto para o profissional e paciente (Husczcz, 2018).

A implantação de consultas farmacêuticas vem proporcionando a realização de um atendimento mais humanizado ao paciente, o que consequentemente permite gerar valorização profissional, maior adesão e eficácia aos tratamentos recomendados (LOPES, 2017).

O farmacêutico pode realizar indicação farmacêutica, ou seja, indicação de medicamento isento de prescrição (MIP) em caso de comprovada necessidade, caso contrário deve-se realizar o encaminhamento ao médico (Melgarejo, 2019).

"O farmacêutico clínico não veio para tomar o lugar do médico, mas para intermediar a relação entre pacientes e médicos. Somos um elo para agregar qualidade de vida ao paciente" (de Paiva, 2021).

\section{Atenção farmacêutica}

A Atenção Farmacêutica é a criação de serviços de clínica, permitindo ao farmacêutico o gerenciamento integrado da farmacoterapia, gerando um controle mais eficaz das doenças, maior segurança para o paciente e contribuindo para a melhoria na sua qualidade de vida (Husczcz, 2018).

A base da atenção farmacêutica é o paradigma do cuidado ao paciente, onde todos os profissionais envolvidos cooperam entre si, visando o benefício ao paciente (Vasconcelos, 2016).

\section{Assistência farmacêutica}

Assistência farmacêutica (AF), caracterizada como um conjunto de ações, desenvolvidas pelo farmacêutico em conjunto com outros profissionais de saúde, focadas no medicamento (desde sua seleção até a dispensação), voltadas à promoção, à proteção e à recuperação da saúde (Husczcz, 2018).

A Assistência Farmacêutica (AF) são ações voltadas à promoção, proteção e recuperação da saúde, tanto individual como coletivo, tendo o medicamento como insumo essencial e visando o acesso e ao seu uso racional (Carvalho, 2017). 
AF que pode desenvolver vários benefícios aos métodos de tratamentos de saúde para quem faz o uso de medicamentos, obtendo excelentes resultados, por exemplo, no aumento da eficácia do tratamento, na melhoria da qualidade de vida dos pacientes e na diminuição de custos dos serviços de saúde (Pereira, 2017).

Segundo a Política Nacional de Medicamentos (PNM), têm como objetivo principal garantir o acesso da população a medicamentos essenciais de qualidade, promovendo, dessa forma, seu uso racional e, diante disso, entende-se o papel indispensável do farmacêutico como profissional da saúde (Araújo, 2017).

$\mathrm{Na} \mathrm{AF}$, a avaliação é feita por meio da observação de prontuários internos, atenção farmacêutica ou durante a dispensação, buscando informações diretamente com o paciente ou analisando as prescrições medicamentosas (SÁ et al., 2020). Com isso, os problemas relacionados ao uso irracional dos medicamentos podem ser diminuídos, favorecendo assim uma melhor qualidade de vida a aquele paciente (Araújo et al., 2017).

\section{A farmácia clínica no cuidado ao paciente}

A farmácia clínica é a área voltada para o cuidado do paciente que visa à promoção, proteção e recuperação da saúde e prevenção de seus agravos, devido ao uso inadequado de medicamentos (de Lima, 2017).

É uma atribuição clínica do farmacêutico, decorrente do paciente ou de encaminhamento de outro profissional de saúde, é avaliação cuidadosa, seleção da melhor conduta e documentação (prontuário), constitui, conforme Resolução CFF n ${ }^{\circ}$ 585/2013, uma consulta farmacêutica (Lopes, 2017).

O farmacêutico é de fundamental relevância quanto ao acompanhamento farmacoterapêutico, uma vez que o paciente necessita de cuidados e orientação quanto ao uso racional de medicamentos e hábitos de vida adequados para o controle de suas doenças (Melgarejo, 2019).

O serviço de clínica farmacêutica atende às necessidades dos usuários relacionadas aos medicamentos de forma integrada, contínua, segura e efetiva, visando à obtenção de resultados terapêuticos concretos (Husczcz, 2018).

Para detectar reações adversas de medicamentos e prevenir problemas relacionados a medicamentos (PRM) evitáveis, muitos hospitais certificados têm feito esforços para criar Serviços de Farmácia Clínica (SFC) (Okumura, 2016).

As atividades desenvolvidas pelos farmacêuticos clínicos representam um importante papel na promoção do uso racional de medicamentos, assegurando que os pacientes recebam a farmacoterapia adequada, além de minimizar os riscos de desfechos desfavoráveis da farmacoterapia e reduzir os custos (Vasconcelos, 2016).

\section{Metodologia}

Este estudo se caracteriza por uma revisão integrativa. Considera-se uma revisão integrativa de literatura um método que permite a síntese de conhecimento e a incorporação dos resultados de estudos significativos na prática (de Sousa, 2017). O período que compôs a elaboração deste trabalho foi do ano de 2016 a 2021. As informações aqui descritas foram coletadas na BVS (Biblioteca Virtual da Saúde) e Google Acadêmico, utilizando os descritores: "consultório farmacêutico", "consulta clínica", "farmacêutico clínico" e seus respectivos correspondentes em inglês. A partir desses descritores e para a busca dos artigos, através dos filtros das próprias bases de dados, estabeleceram-se os seguintes critérios de inclusão: artigos disponíveis na íntegra, em português e inglês, no período de 2016 a 2021 com acesso gratuito e que tivessem afinidade com a temática. Identificaram-se no total 40 publicações, após aplicação dos critérios de inclusão, foram excluídos 20 estudos identificados por meio de pesquisas em outras fontes de dados, permanecendo 20 estudos. Os resultados apresentados acima foram dispostos ao Fluxograma 1. 
Fluxograma 1: Dados dos artigos utilizados:
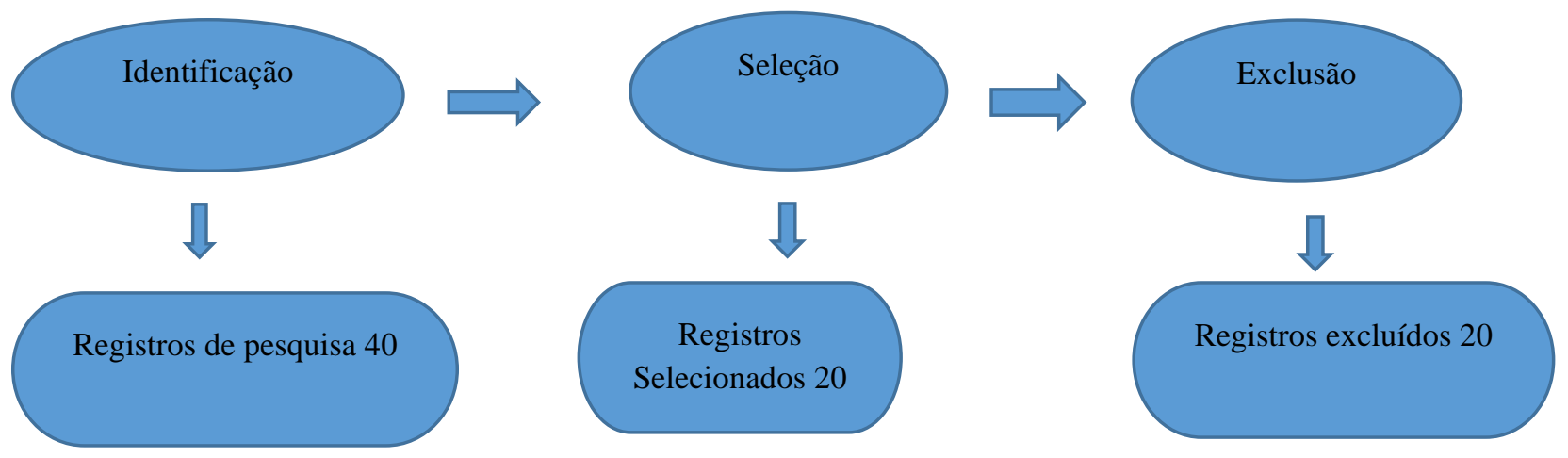

Fonte: Autores, Redenção - PA (2021).

Para a identificação dos documentos de interesse para estudos foi observado o título e o resumo dos artigos, elegendo os utilizados. Foram desconsiderados todos aqueles que não contemplavam o tema.

\section{Resultados e Discussão}

Entre os 10 artigos selecionados, 4 são revisões bibliográficas e 6 de revisões descritivas/experimentais. O perfil e características dos estudos estão apresentados na Tabela 1.

Tabela 1: Principais estudos sobre o perfil das consultas clínicas em consultórios farmacêuticos.

\begin{tabular}{|c|c|c|c|}
\hline ARTIGO & OBJETIVO & $\begin{array}{c}\text { DESENHO } \\
\text { DO ESTUDO } \\
\end{array}$ & CONSIDERAÇÕES FINAIS \\
\hline $\begin{array}{l}\text { LOPES, D. A. } \\
\text { M. G., \& Cruz, } \\
\text { F. O. (2016) }\end{array}$ & $\begin{array}{l}\text { Realizar um estudo sobre o } \\
\text { consultório farmacêutico e } \\
\text { sua importante contribuição } \\
\text { para à saúde da população. }\end{array}$ & $\begin{array}{l}\text { Revisão } \\
\text { Descritiva/Ex } \\
\text { perimental }\end{array}$ & $\begin{array}{l}\text { Por meio deste estudo executou-se um repertório específico de } \\
\text { literaturas sobre o tema abordado, com o propósito de realizar uma } \\
\text { investigação qualitativa sobre o consultório farmacêutico. Além } \\
\text { disso, também se buscou fazer um levantamento sobre esses } \\
\text { possíveis problemas e como a assistência farmacêutica pode } \\
\text { colaborar para suavizar e/ou reduzir os problemas com } \\
\text { medicamentos, tendo como base a concepção dos autores } \\
\text { analisados. Acerca das dúvidas surgidas sobre a eficácia da } \\
\text { assistência farmacêutica na diminuição de problemas com } \\
\text { medicamentos, na percepção dos teóricos estudados, as respostas } \\
\text { obtidas foram de que o acompanhamento e auxilio visando uma } \\
\text { utilização consciente de medicamentos, conforme já exposto uma } \\
\text { melhoria na qualidade de vida das pessoas, bem como uma redução } \\
\text { nos problemas decorridos pelo mau uso dos fármacos. A Atenção } \\
\text { Farmacêutica é uma prática farmacêutica importante para o sistema } \\
\text { de saúde brasileiro, já que pode beneficiar o paciente no uso } \\
\text { racional e eficaz de medicamentos. }\end{array}$ \\
\hline $\begin{array}{lr}\text { Okumura, } & \text { L. } \\
\text { M., da Silva, D. } \\
\text { M., } \\
\text { Comarella, } & \text { L. } \\
(2016) . & \end{array}$ & $\begin{array}{l}\text { Discutir o efeito da } \\
\text { implantação de um SFC à } \\
\text { beira do leito em uma } \\
\text { Unidade de Cuidados } \\
\text { Intensivos Pediátricos (UCIP) } \\
\text { brasileira. }\end{array}$ & $\begin{array}{l}\text { Revisão } \\
\text { Descritiva/exp } \\
\text { erimental }\end{array}$ & $\begin{array}{l}\text { Reações adversas a medicamentos devido a PRM evitáveis podem } \\
\text { ser prevenidas por SFC em uma colaboração multifacetada com } \\
\text { outros profissionais de saúde. Tais problemas podem ser evitados } \\
\text { por meio de estratégias ativas e baseadas em evidências para } \\
\text { reduzir a morbidade relacionada a medicamentos. }\end{array}$ \\
\hline $\begin{array}{l}\text { PEREIRA, A. } \\
\text { V. L. (2017). }\end{array}$ & $\begin{array}{l}\text { Avaliar a assistência } \\
\text { farmacêutica para } \\
\text { oncológico. }\end{array}$ & $\begin{array}{l}\text { Revisão } \\
\text { Bibliográfica }\end{array}$ & $\begin{array}{l}\text { Através desta pesquisa foi possível identificar a importância do } \\
\text { farmacêutico como componente da equipe de saúde na oncologia e } \\
\text { é responsável pela assistência farmacêutica para o paciente } \\
\text { oncológico, uma ação considerada como um conjunto de vários } \\
\text { procedimentos essências para promoção, prevenção e recuperação } \\
\text { a saúde do indivíduo, com uma melhora qualidade de vida. }\end{array}$ \\
\hline $\begin{array}{l}\text { Mesquita, E. T., } \\
\text { Jorge, A. J. L., } \\
\text { Rabelo, L. M., } \\
\text { \& Souza, C. V. } \\
(2017)\end{array}$ & $\begin{array}{l}\text { O objetivo desta revisão foi } \\
\text { discutir as principais causas } \\
\text { de hospitalização, seu } \\
\text { impacto na evolução da } \\
\text { insuficiência cardíaca e as }\end{array}$ & $\begin{array}{l}\text { Revisão } \\
\text { Bibliográfica }\end{array}$ & $\begin{array}{l}\text { A abordagem multidisciplinar associada à implementação de boas } \\
\text { práticas embasadas em evidências científicas pode reduzir o risco } \\
\text { de internação. A aplicação dessas rotinas, que comprovadamente } \\
\text { reduzem a internação hospitalar, deveria ser feita nos hospitais } \\
\text { brasileiros. }\end{array}$ \\
\hline
\end{tabular}




\begin{tabular}{|c|c|c|c|}
\hline & $\begin{array}{l}\text { estratégias que podem ser } \\
\text { adotadas para sua redução. }\end{array}$ & & \\
\hline $\begin{array}{l}\text { Carvalho, M. } \\
\text { N., Álvares, J., } \\
\text { Costa, K. S., } \\
\text { Guerra Junior, } \\
\text { A. A., Acurcio, } \\
\text { F. D. A., Costa, } \\
\text { E. A., ․ \& } \\
\text { Leite, S. N. } \\
(2017) .\end{array}$ & $\begin{array}{l}\text { Caracterizar a força de } \\
\text { trabalho da assistência } \\
\text { farmacêutica na atenção } \\
\text { básica do Sistema Único de } \\
\text { Saúde. }\end{array}$ & $\begin{array}{l}\text { Revisão } \\
\text { Descritiva/Ex } \\
\text { perimental }\end{array}$ & $\begin{array}{l}\text { A profissionalização das funções de gestão municipal na atenção } \\
\text { básica é uma conquista na organização da força de trabalho da } \\
\text { assistência farmacêutica. No entanto, há importantes deficiências } \\
\text { na composição da força de trabalho nas unidades de dispensação de } \\
\text { medicamentos que devem comprometer a qualidade do uso dos } \\
\text { medicamentos e seus resultados na saúde da população. }\end{array}$ \\
\hline $\begin{array}{l}\text { Huszcz, R. S., } \\
\text { del Olmo Sato, } \\
\text { M., \& Santiago, } \\
\text { R. M. (2018). }\end{array}$ & $\begin{array}{l}\text { Descrever sobre o perfil dos } \\
\text { pacientes atendidos nas } \\
\text { consultas farmacêuticas assim } \\
\text { como, as intervenções } \\
\text { realizadas, bem como a } \\
\text { satisfação do paciente em } \\
\text { relação as consultas } \\
\text { farmacêuticas. }\end{array}$ & $\begin{array}{l}\text { Revisão } \\
\text { Descritiva/Ex } \\
\text { perimental. }\end{array}$ & $\begin{array}{l}\text { A consulta farmacêutica apresentou resultados positivos em relação } \\
\text { ao tratamento do paciente assim como os pacientes atendidos } \\
\text { mostraram-se satisfeitos com a consulta farmacêutica. }\end{array}$ \\
\hline $\begin{array}{l}\text { Reis, W. C. T., } \\
\text { dos } \quad \text { Santos } \\
\text { Bernardo, C., de } \\
\text { Souza, T. T., de } \\
\text { Fátima Bonetti, } \\
\text { A., \& Favero, } \\
\text { M. L. D. }(2018) \text {. }\end{array}$ & $\begin{array}{l}\text { Avaliar o impacto do serviço } \\
\text { de farmácia clínica em } \\
\text { pacientes polifarmáticos } \\
\text { atendidos em ambulatório de } \\
\text { hospital de grande porte. }\end{array}$ & $\begin{array}{l}\text { Revisão } \\
\text { Integrativa }\end{array}$ & $\begin{array}{l}\text { O acompanhamento ambulatorial por farmacêutico clínico tem } \\
\text { potencial para obtenção de resultados positivos na saúde do } \\
\text { paciente. }\end{array}$ \\
\hline $\begin{array}{l}\text { Melgarejo, A. P. } \\
\text { (2019). }\end{array}$ & $\begin{array}{l}\text { Realizar, na prática, as } \\
\text { atribuições pertinentes ao } \\
\text { farmacêutico em um ambiente } \\
\text { público e carente de } \\
\text { informações farmacológicas e } \\
\text { não farmacológicas, as quais } \\
\text { podem melhorar a qualidade } \\
\text { de vida dos pacientes. }\end{array}$ & $\begin{array}{l}\text { Descritiva/Ex } \\
\text { perimental }\end{array}$ & $\begin{array}{l}\text { Diante disso, pode-se entender que os cuidados farmacêuticos são } \\
\text { imprescindíveis, tanto quanto ao acesso ao medicamento, quanto ao } \\
\text { uso racional, pois juntamente com outros profissionais de saúde, o } \\
\text { farmacêutico pode realizar o acompanhamento da terapia e realizar } \\
\text { orientações farmacológicas e não farmacológicas que poderão } \\
\text { melhorar a adesão ao tratamento bem como a qualidade de vida do } \\
\text { paciente. }\end{array}$ \\
\hline
\end{tabular}

Fonte: Autores (2021).

Na Tabela 1 os autores em âmbito geral relatam a importância do consultório farmacêutico no atendimento à consulta farmacêutica, nesse momento o paciente tem a oportunidade de criar um vínculo com o profissional viabilizando a garantia da segurança e eficácia do tratamento e da adesão à farmacoterapia, relatando as suas dificuldades e sucessos com o tratamento.

Nos dias atuais os consultórios médicos estão sendo altamente procurados dificultando o acesso do paciente e cuidador ao profissional em seus consultórios, diante desse agravamento da condição clínica na saúde da população os consultórios farmacêuticos se tornaram uma solução rápida e viável para solucionar na medida do possível seus problemas de saúde.

A implantação dos consultórios é uma forma rápida e garante muitos retornos benéficos à população, sejam esses consultórios estabelecidos em farmácias, drogarias, hospitais o acesso do paciente ao profissional farmacêutico é imprescindível na condução de seu tratamento (de Paiva, 2021).

As consultas farmacêuticas vêm se tornando a principal escolha entre os pacientes pois permitem a eles a exposição dos seus problemas de modo seguro, privativo e eficaz construindo de modo satisfatório a interação paciente/farmacêutico/médico, se tornando um elo entre os mesmos trabalhando a saúde de modo preventivo reduzindo o surgimento de outras patologias e/ou o agravamento das pré-existentes (Lopes, 2016).

Carvalho (2017) nos mostra que a assistência farmacêutica (ação voltada ao cuidado dos pacientes em uma comunidade se faz necessária e quando possível a utilização da atenção farmacêutica dentro desse mesmo local tratando em particular o caso mencionado, dando uma atenção exclusiva à demanda daquele paciente específico em especial. 
Através da atenção e da assistência farmacêutica o clínico através da consulta transmite ao paciente informações capazes de reduzir ou extinguir os Problemas Relacionados aos Medicamentos (PRM's), isso é um processo que exige comprometimento de ambas as partes, pois o profissional se compromete a entender a terapia aplicada e o paciente a realizar da melhor forma possível num processo otimizado que pode a qualquer momento ser revisto e ajustados às necessidades (Okumura, 2016).

Os pacientes que mais procuram os consultórios farmacêuticos são em sua grande maioria idosos que praticam polifarmácia e enxergam o farmacêutico como um grande auxílio no entendimento no momento da administração dos seus medicamentos que necessitam ser organizados de modo que o cuidador ou o próprio paciente consiga identificar e realizar a ingestão sem nenhuma dúvida ou complicação (Huszcz, 2018).

Os pacientes com casos de doenças crônicas são também frequentadores assíduos das consultas farmacêuticas para fazer o acompanhamento farmacoterapêutico e verificar se o mesmo está conseguindo manter estáveis os seus problemas tratados alcançando assim uma vida normal diante da realidade de sua patologia (de Sá, 2020).

De acordo com Pereira (2016), durante as consultas farmacêuticas os pacientes e seus cuidadores tem a oportunidade de esclarecer as suas principais dúvidas e inseguranças em relação ao seu tratamento tornando dessa forma uma maneira mais suave na condução da sua terapia que muitas vezes pode ser longa e sofrida, em alguns momentos o desespero faz com que o paciente desista e o farmacêutico com todo o cuidado faz a mediação entre o saber clínico e a humanização intensificando os cuidados com os seus pacientes especiais.

Nos ambulatórios os pacientes que recebem o acompanhamento do farmacêutico têm grande adesão ao tratamento o que gera uma saída mais rápida dos hospitais e o retorno com maior qualidade de vida à sua vida normal (Reis, 2018).

O farmacêutico mesmo sendo o profissional de mais fácil acesso enfrenta ainda alguns obstáculos em relação aos pacientes que se automedicam e consequentemente acabam por usar em alguns casos medicamentos desnecessários ou que tragam alguma reação adversa (Melgarejo, 2019) em consequência do uso incorreto, tais ações podem levar ao aumento nos casos de internação e sobrecarregando os hospitais, aumentando os gastos com internações e desgastando ainda mais o sistema de cuidados emergenciais ao paciente (Mesquita, 2017).

\section{Considerações Finais}

Através do presente artigo, fora possível analisar o perfil das consultas clínicas em consultórios farmacêuticos, levando-se em consideração que diversos aspectos influenciam no comportamento das pessoas, tais como intimidade junto ao médico, vergonha, dificuldade de se expressar entre outros, assim deve se fundamentar nas necessidades de saúde da população, em especial dos segmentos socialmente mais vulneráveis, na busca da equidade e racionalidade no acesso à atenção à saúde e a tratamentos.

Dessa forma pode se perceber que a Atenção Farmacêutica é a interação direta do farmacêutico com as pessoas, visando uma farmacoterapia mais racional e uma obtenção de resultados que sejam definidos e mensuráveis, voltados para a melhoria da qualidade de vida.

Ainda é possível salientar, o quanto o farmacêutico ocupa um papel-chave na Assistência Farmacêutica, pois é o único profissional da equipe de saúde que tem sua formação técnico-científica fundamentada na articulação de conhecimentos das áreas biológicas e exatas.

Portanto, a Assistência Farmacêutica tem ainda um grande caminho a ser percorrido. A necessidade de tratar esse tema com a devida responsabilidade é imprescindível. Para isso, a qualificação do profissional farmacêutico, assumindo suas funções de gestor do ciclo da Assistência Farmacêutica, assim como seu papel na atenção farmacêutica, é determinante. 
Os consultórios clínicos em local privativo e de fácil acesso serão um meio fundamental de integrar a prática clínica e o paciente fornecendo meios para o farmacêutico adquirir as informações necessárias através da anamnese relatadas em prontuário e garantindo a segurança e o cuidado ao paciente fazendo a diferença na vida dos mesmos.

\section{Agradecimentos}

Agradeço minha orientadora que me proporcionou todas as ferramentas para alcançar meu sucesso! Os professores são os pilares da nossa sociedade porque são a base para todas as profissões! Todo o meu carinho e gratidão àqueles que ensinam muito mais que teorias, e sim que nos preparam também para a vida. (Rafael Galvan Luiz)

\section{Referências}

Araújo, S. Q., Costa, K. S., Luiza, V. L., Lavras, C., Santana, E. A., \& Tavares, N. U. L. (2017). Organização dos serviços farmacêuticos no Sistema Único de Saúde em regiões de saúde. Ciência \& Saúde Coletiva, 22, 1181-1191. https://doi.org/10.1590/1413-81232017224.27042016

Bouças, E. (2017). Implicações do processo de acreditação em serviços de farmácia de hospitais privados sob a perspectiva da qualidade da assistência farmacêutica: um estudo de caso.

Canto, V. B. D. (2016). Implementação de um Serviço de Clínica Farmacêutica em uma Unidade Básica de Saúde (UBS) do município de Florianópolis: Um Relato de Experiência.

Carvalho, M. N., Álvares, J., Costa, K. S., Guerra Junior, A. A., Acurcio, F. D. A., Costa, E. A., \& Leite, S. N. (2017). Força de trabalho na assistência farmacêutica da atenção básica do SUS, Brasil. Revista de Saúde Pública, 51, 16s.

De Lima, É. D., Da Silva, R. G., Ricieri, M. C. \& Blatt, C. R. (2017). Farmácia clínica em ambiente hospitalar: enfoque no registro das atividades. Revista Brasileira de Farmácia Hospitalar e Serviços de Saúde, 8(4). https://doi.org/10.30968/rbfhss.2017.084.004

de Paiva, L. A., \& dos Anjos, D. M. (2021). Aspectos relacionados a implementação de consultório farmacêutico em farmácias de rede privada. Brazilian Applied Science Review, 5(3), 1567-1585. DOI:10.34115/basrv5n

de Sá, M. G. F., da Silva Lima, V., de Sá Leal, M. S., \& Lima, C. G. (2020). Avaliação da compreensão e adesão da terapia medicamentosa de pacientes diabéticos assistidos no município de Verdejante-PE. Brazilian Journal of Health Review, 3(4), 7225-7235.

de Sousa, L. M. M., Marques-Vieira, C. M. A., Severino, S. S. P., \& Antunes, A. V. (2017). A metodologia de revisão integrativa da literatura em enfermagem. $N^{o} 21$ Série 2-Novembro 2017, 17.

Huszcz, R. S., del Olmo Sato, M., \& Santiago, R. M. (2018). Consultório farmacêutico: atuação do farmacêutico no SUS. Revista Saúde e Desenvolvimento, 12(10), 144-159.

Lima, J. R., Prata, S. S. B., da Silva, L. O., da Silva, A. H. M., de Carvalho, W. C. P., Cabral, P. C. R. \& Costa, R. D. C. A. (2021). Proposta de consultório farmacêutico especializado para tuberculose. Mostra de Inovação e Tecnologia São Lucas (2763-5953), 2(1), 248-50.

LOPES, D. A. M. G., \& Cruz, F. O. Atenção farmacêutica e consultórios farmacêuticos. Revista das Faculdades Oswaldo Cruz. Ed, 16.

Melgarejo, A. P. (2019). Consultório farmacêutico: atuação e contribuição do farmacêutico no acompanhamento de pacientes com problemas de saúde.

Mesquita, E. T., Jorge, A. J. L., Rabelo, L. M., \& Souza, C. V. (2017). Entendendo a hospitalização em pacientes com insuficiência cardíaca. International Journal of Cardiovascular Sciences, 30, 81-90.

Okumura, L. M., da Silva, D. M., \& Comarella, L. (2016). Relação entre o uso seguro de medicamentos e serviços de farmácia clínica em Unidades de Cuidados Intensivos Pediátricos. Revista Paulista de Pediatria, 34, 397-402. http://dx.doi.org/10.1016/j.rppede.2016.04.001

Pereira, A. V. L. (2017). Assistência farmacêutica para o paciente oncológico.

Reis, W. C. T., dos Santos Bernardo, C., de Souza, T. T., de Fátima Bonetti, A., \& Favero, M. L. D. (2018). Impacto da consulta farmacêutica em pacientes polimedicados com alto risco cardiovascular. Revista Brasileira de Farmácia Hospitalar e Serviços de Saúde, 9(2).10.30968/rbfhss.2018.092.003

Rover, M. R. M., Peláez, C. M. V., Faraco, E. B., Farias, M. R., \& Leite, S. N. (2017). Avaliação da capacidade de gestão do componente especializado da assistência farmacêutica. Ciência \& Saúde Coletiva, 22, 2487-2499. https://doi.org/10.1590/1413-81232017228.01602017

Silva, M. J. S. D. (2018). Atenção oncológica e assistência farmacêutica no Sistema Único de Saúde (Doctoral dissertation).

Silva Júnior, J. A. D. (2019). Atenção farmacêutica no uso racional de medicamentos como estratégia na promoção da saúde aos grupos pediátricos e geriátricos: Uma revisão integrativa.

Vasconcelos, T. Y. L., Cangussú, I. M., Mesquita, R. J., Marques, F. V. B. S., \& Nascimento, A. A. (2016). A farmácia clínica no âmbito da farmácia magistral. J Appl Pharm Sci [Internet], 3, 4-6. 